\title{
Microglia and macrophages in human glioblastomas: A morphological and immunohistochemical study
}

\author{
MAGNUS KVISTEN $^{1}$, VILDE E. MIKKELSEN ${ }^{1}$, ANNE LINE STENSJØEN ${ }^{2}$, OLE SOLHEIM ${ }^{2-4}$, \\ JOHANNES VAN DER WANT ${ }^{1}$ and SVERRE H. TORP ${ }^{1,5}$
}

\author{
${ }^{1}$ Department of Clinical and Molecular Medicine, Faculty of Medicine and Health Sciences, \\ NTNU-Norwegian University of Science and Technology, NO-7491 Trondheim; ${ }^{2}$ Department of Neurosurgery, \\ St. Olavs University Hospital, NO-7006 Trondheim; ${ }^{3}$ Department of Neuromedicine and Movement Science, \\ Faculty of Medicine, NTNU-Norwegian University of Science and Technology, NO-7491 Trondheim; \\ ${ }^{4}$ National Competence Centre for Ultrasound and Image Guided Therapy, St. Olavs Hospital; \\ ${ }^{5}$ Department of Pathology, St. Olavs Hospital, NO-7006 Trondheim, Norway
}

Received October 25, 2018; Accepted March 5, 2019

DOI: $10.3892 /$ mco.2019.1856

\begin{abstract}
Glioblastomas (GBMs), a type of highly malignant brain tumour, contain various macrophages/microglia that are known as tumour-associated macrophages (TAMs). These TAMs have various roles in tumour biology. Histopathological aspects of TAMs and associations with tumour growth assessed by magnetic resonance imaging (MRI) are poorly described. In the present study, 16 patients that had sufficient tumour tissue and histological hallmarks were examined. The tumours were classified as either slow- $(n=7)$ or fast-growing $(n=9)$ based on the segmented tumour volumes from MRI scans taken at diagnosis and preoperatively. Using cluster of differentiation (CD)68 and ionized calcium-binding adaptor molecule 1 (Iba1) antibodies, the number, morphology, localization and distribution of TAMs in the GBM tissue were studied. TAMs were significantly more immunoreactive for anti-Iba1 (TAMs ${ }^{\mathrm{Iba}}$ ) compared with anti-CD68 (TAMs ${ }^{\mathrm{CD} 68}$; $\mathrm{P}<0.001)$. In central tumour areas and around vessels in the infiltration zone there were more $\mathrm{TAMs}^{\mathrm{CD} 68}$ in slow-growing tumours $(\mathrm{P}=0.003$ and $\mathrm{P}=0.025$, respectively). Central tumour areas contained more TAMs compared with the infiltration zone $\left(\mathrm{P}=0.001\right.$ for TAMs ${ }^{\mathrm{CD} 68}$ and $\mathrm{P}<0.001$ for $\left.\mathrm{TAMs}^{\mathrm{Iba1}}\right)$.
\end{abstract}

Correspondence to: Professor Sverre H. Torp, Department of Pathology, St. Olavs Hospital, Erling Skjalgssons gate 1, NO-7006 Trondheim, Norway

E-mail: sverre.torp@ntnu.no

Abbreviations: GBM, glioblastoma multiforme; TAMs, tumour-associated macrophages; $\mathrm{TAMs}^{\mathrm{CD} 68}$, tumour-associated macrophages immunoreactive for CD68 antibody; TAMs ${ }^{\text {Ibal }}$, tumour-associated macrophages immunoreactive for Ibal antibody

Key words: glioblastoma, microglia, tumour-associated macrophages, macrophages, immunohistochemistry
The majority of TAMs exhibited a ramified phenotype in the infiltration zone, whereas central TAMs were mostly amoeboid. TAMs were present in high numbers in most regions of the tumour, whereas there were few in necrotic areas. In conclusion, the present study demonstrated and confirmed that the high numbers of TAMs in GBMs assume a range of morphologies consistent with various activation states, and that slow-growing GBMs seem to contain a TAM-population different to their fast-growing counterparts.

\section{Introduction}

Glioblastomas (GBMs) are highly malignant brain tumours with a poor prognosis, despite aggressive surgical and oncological treatments. They are genetically and histopathologically highly heterogeneous $(1,2)$. In addition to malignant astrocytes, there are also large numbers of microglia/macrophages, known as tumour-associated macrophages (TAMs), which can account for up to $30-50 \%$ of the total tumour cell mass in human GBMs (3). Microglial proliferation or differentiation of macrophages from blood-borne precursors contribute to TAM accumulation (4). The influence of TAMs on GBM growth seems to be multifaceted and dependent on the polarization of TAMs; thus, TAMs are potential therapeutic targets (5). TAM activation is complex and comprises two dynamic states: M1 (pro-inflammatory) or M2 (anti-inflammatory), which translates to tumour suppressive (M1) or tumour supportive (M2). These activities are closely linked to glioma maintenance and progression $(3,6,7)$. However, this categorization of TAMs is debatable and based on in vitro studies (5).

In GBMs, TAMs can assume different morphologies (8), i.e. ramified and amoeboid appearances related to various functional states (9). The ramified phenotype, which is only expressed by microglia, is considered typical for a 'resting' phase; whereas, the amoeboid phenotype that is expressed by both microglia and macrophages, is associated with a more active state (10). Much research in this field is based on cell lines and animal studies. Therefore, few studies have explored 
the distribution and frequency of TAMs in relation to the growth of human GBMs.

Recently, the pretreatment growth dynamics of a cohort of 106 GBMs were estimated using tumour volume segmentations from two contrast-enhancing T1-weighted MRIs, from diagnostic and preoperative scans (11). The GBMs were divided into two groups, slow and fast-growing tumours, and the former was determined to be a significant predictor of long term survival (12). From the original pretreatment 106 GBMs we selected 16 cases that contained sufficient tissue to study MRI-estimated tumour growth in relation to histopathological TAM distribution. TAMs were classified using CD68 and Iba1, two common macrophage/microglia markers (13). The aims of this study were to examine the morphology, distribution, and density of TAMs in GBM tissue by means of immunohistochemistry using CD68 and Iba1 in relation to the MRI-estimated slow- and fast-growth properties.

\section{Materials and methods}

The GBM tissues used in this paper were from sixteen patients from a previous study that estimated pretreatment growth rates in 106 adult GBM patients operated at St. Olavs University Hospital, Trondheim, Norway between January 2004 and May 2014 (11). From the 106 initial GBMs, 16 had sufficient tissue to assess the distribution of TAMs in various locations (i.e. central and peripheral areas). All cases were previously shown to be GFAP-immunoreactive and IDH1 R132H immunonegative (12). Clinical data were extracted from electronic medical records at St. Olavs University Hospital.

Since all patients underwent at least two MRI examinations (one at the time of diagnosis and one taken before operation), MRI-estimated, pretreatment GBM growth could be estimated based on manual segmentation of both tumour volumes (11). A detailed description of the segmentation, estimation of growth rates, and the categorization into two growth groups has been reported elsewhere $(11,12)$. Seven of the included tumours displayed slow growth and nine displayed fast growth. Routine haematoxylin and eosin $(\mathrm{H} \& \mathrm{E})$ stained $5 \mu \mathrm{m}$ paraffin tissue sections were revised and classified according to 2016-WHO-criteria. Sections were incubated with the polyclonal antibody Iba1 (ab5076, goat polyclonal; Abcam Inc., Cambridge MA, USA), dilution 1:500, and CD68 (M 0814, mouse clone KP1; Dako, Glostrup, Denmark), dilution 1:6,000, for $30 \mathrm{~min}$ at room temperature. Standard immunohistochemical procedures were followed using an automated staining unit (Dako Techmate 500). Human spleen served as the positive control, and the primary antibody was omitted in the negative control.

We distinguished the following areas: The infiltration zone, defined as the periphery of the tumour containing both normal brain and tumour cells and central areas with emphasis on necrosis, pseudopalisades, and perivascular areas. Histologic assessment of the localization and distribution of TAMs and their morphology was performed throughout the tumour tissue, as well as the number of TAMs. Areas with the highest density of labelled TAMs were then identified using the 10x objective. The amount of immunoreactive cells was then semi-quantitatively categorized in high power fields (40x objective) using the following rating system for the amounts of immunoreactive cells: Low $(+, \sim 0-10 \%$ of the cells were positive), moderate $(++$, $\sim 10-50 \%$ of the cells were positive), and high (+++, $>50 \%$ of the cells were positive). TAM morphology was classified as a dominating ramified or amoeboid phenotype (Fig. 1). Cells with a so-called 'bushy' phenotype were classified as ramified TAMs (14). The microscopic analyses were performed by MK, VEM, and an experienced neuropathologist (SHT) using a Nikon Eclipse 80i microscope.

Statistical analysis was performed using SPSS version 22, and comparisons between groups were based on the Mann-Whitney U test or Wilcoxon signed rank test. Based on Bonferroni correction for 20 tests, P-values $<0.0025$ were considered statistically significant. This project was approved by the Regional Ethics Committee (Central) as part of a larger project (references 2011/974 and 2013/1348) and adhered with the Declaration of Helsinki.

\section{Results}

Sixteen adult GBM patients with a median age of 62 years (range 50-79) were included in this study, five females and 11 males. Among these patient's tumours, nine were fast-growing and seven were slow-growing. The distribution of TAMs identified with CD68 and Ibal is shown in Table I; pseudopalisading regions are not included, as they were observed in only 12 of the cases. The infiltration zones showed a dispersed distribution of TAMs compared with central tumour areas.

In central parts of the tumour, there were statistically higher numbers of TAMs compared with the periphery for both antibodies $\left(\mathrm{P}=0.001\right.$ for $\mathrm{TAMs}{ }^{\mathrm{CD} 68}$ and $\mathrm{P}<0.001$ for TAMs $^{\text {Ibal }}$ )(Table II). There was both diffuse as well as condensed infiltration of TAMs in various histological compartments, such as the perivascular spaces, pseudopalisades, and in areas between larger vessels and necrotic areas (Fig. 2). However, there were few TAMs within necrotic areas. In central tumour areas, most TAMs were amoeboid.

Slow- and fast-growing tumours contained different quantities of TAMs ${ }^{\mathrm{CD} 68}$, whereas no such difference was seen with TAMs $^{\text {Ibal }}$. Slow-growing tumours contained more perivascular TAMs ${ }^{\mathrm{CD} 68}$ in the infiltration zone and more TAMs ${ }^{\mathrm{CD} 68}$ in central areas $(\mathrm{P}=0.003$ and $\mathrm{P}=0.025$ respectively) (Fig. 3); however, these results did not reach the significance level set by the Bonferroni correction $(\mathrm{P}<0.0025)$. Comparisons between slow- and fast-growing tumours are summarized in Table III.

\section{Discussion}

In this study, we investigated the histopathological aspects of TAMs in human GBMs with emphasis on the number, distribution and morphology of Iba1 and CD68 immunoreactive TAMs, as well as the relationship with tumour growth estimated from MRI scans. In specific tumour regions, there were more TAMs ${ }^{\mathrm{CD} 68}$ in the slow-growing GBMs, while the quantities of TAMs ${ }^{\mathrm{Iba} 1}$ were similar in slow- and fast-growing tumours. There were significantly more TAMs $^{\text {Iba1 }}$ than TAMs ${ }^{\mathrm{CD} 68}$. The lbal antibody reacts with an ionized calcium-binding protein typical for both resting and activated microglia/macrophages, whereas anti-CD68 labels lysosomal membranes commonly found in these cells (9). In 

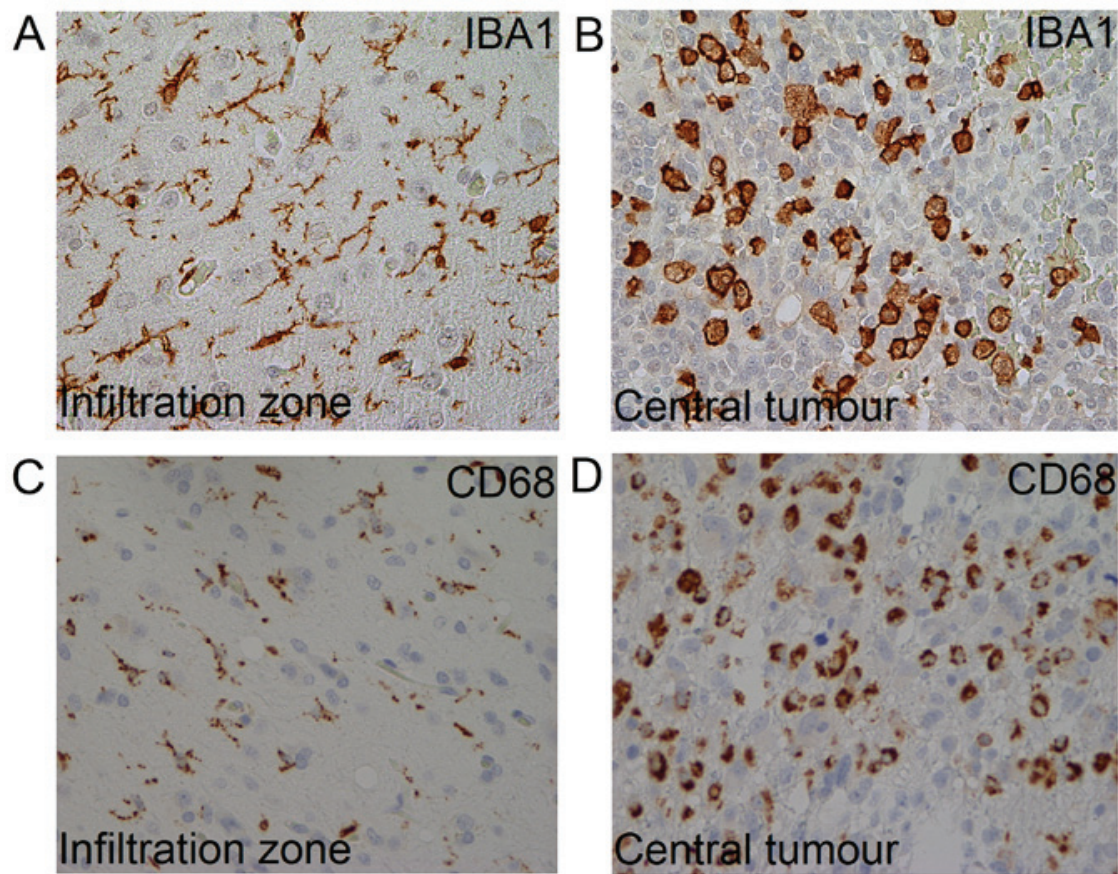

Figure 1. Central and peripheral regions of GBM. (A and B) Iba1 and (C and D) CD68 immunostainings illustrate the different phenotypes, and the numbers of TAMs in the (A and C) infiltration zones and (B and D) central areas. Central tumour areas contained mostly amoeboid TAMs; whereas, TAMs in the infiltration zones displayed a ramified phenotype. In addition, there were more TAMs in central tumour areas compared with infiltration zones (original magnification, x200). GBM, glioblastoma; TAMs, tumour-associated macrophages.

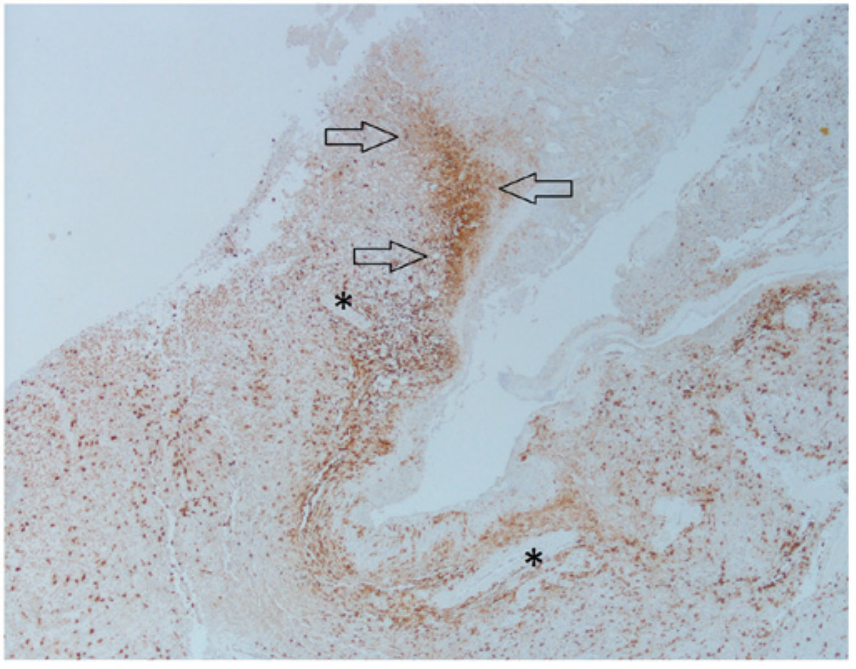

Figure 2. TAMs ${ }^{\mathrm{Ibal}}$ between vessels and necrotic areas. Large numbers of TAMs $^{\text {Ibal }}$ can be observed near vessels (asterisks) and extending into the adjacent necrotic area (arrows) that show few vessels, suggesting a migrant path originating from vessels and heading to necrosis (Fig. 3). The section is from a fast-growing tumour (original magnification, $\mathrm{x} 40$ ). TAMs ${ }^{\mathrm{Iba} 1}$, Iba1-immunoreactive tumour-associated macrophages.

general, these two antibodies are regarded as pan-markers of TAMs $(14,15)$. There are, however, certain differences between them, as Ibal seems to stain more widely (including more of the activation states) than CD68 (16). As there were no differences in TAMs ${ }^{\text {Ibal }}$ between slow and fast-growing tumours, it seems that the absolute number of TAMs is not an essential factor in GBM growth.

Accordingly, the phenotypes and activation states of TAMs may be more relevant. Ramified TAMs dominated the peripheral parts of the tumour, in contrast to the central areas where TAMs mainly had an amoeboid phenotype. TAMs can be simply categorized as classically activated M1 (pro-inflammation/anti-tumour) and alternatively activated M2 (anti-inflammatory/pro-tumour) (5). Actually, there are various antibodies reactive against different epitopes and functional states of TAMs $(9,15)$, and currently no single reliable immunohistochemical marker exists for TAMs. For that reason, standardized markers for histological immunophenotyping of TAMs are needed. Nevertheless, as CD68 is highly expressed among TAMs in the M1 state $(16,17)$, our finding of many $\mathrm{TAMs}^{\mathrm{CD} 68}$ in the periphery of slow-growing GBMs suggests there are growth inhibitory effects of these cells in this part of the tumour that may serve as a potential target for therapy.

To study the TAM morphology, we selected sections of GBM cases with sufficient tumour tissue to display the whole transition from central tumour to the infiltration zone. We observed a gradual increase in the number of TAMs toward the tumour centre. Further, there was a transition, via hybrid forms, of TAMs with a ramified morphology in the periphery to the dominant amoeboid phenotype in central areas. Such a pattern has been described by others $(9,16)$, however, not in a setting as our study, and supports that TAMs can have various phenotypes and activation states throughout the tumour $(10,18,19)$. Since ramified and amoeboid forms of TAMs are linked to low- and high activation states, respectively (9), these observations support a gradual increase in activated TAMs toward the tumour centre. Accordingly, this variable and dynamic TAM population in human GBMs may have various impacts on the biology in different parts of the tumour, and may explain the discrepant effects on TAMs on survival in human GBMs (20). 
Table I. Amount of TAMs ${ }^{\text {Ibal }}$ and $\mathrm{TAMs}{ }^{\mathrm{CD} 68}$ in slow- and fast-growing glioblastomas.

\begin{tabular}{|c|c|c|c|c|c|c|}
\hline \multirow[b]{2}{*}{ Location } & \multicolumn{3}{|c|}{ Slow growth $(n=7)$} & \multicolumn{3}{|c|}{ Fast growth $(\mathrm{n}=9)$} \\
\hline & $\begin{array}{l}\text { Low } \\
\text { amount } \\
+(\%)\end{array}$ & $\begin{array}{c}\text { Moderate } \\
\text { amount } \\
++(\%)\end{array}$ & $\begin{array}{c}\text { High } \\
\text { amount } \\
+++(\%)\end{array}$ & $\begin{array}{l}\text { Low } \\
\text { amount } \\
+(\%)\end{array}$ & $\begin{array}{c}\text { Moderate } \\
\text { amount } \\
++(\%)\end{array}$ & $\begin{array}{c}\text { High } \\
\text { amount } \\
+++(\%)\end{array}$ \\
\hline \multicolumn{7}{|c|}{ Infiltration zone } \\
\hline TAM ${ }^{\mathrm{Iba} 1}$ & $0 / 7(0)$ & 7/7 (100) & $0 / 7(0)$ & $1 / 9(11)$ & $7 / 9(78)$ & $1 / 9(11)$ \\
\hline $\mathrm{TAM}^{\mathrm{CD} 68}$ & $7 / 7(100)$ & $0 / 7(0)$ & $0 / 7(0)$ & $8 / 9(89)$ & $1 / 9(11)$ & $0 / 9(0)$ \\
\hline \multicolumn{7}{|c|}{ Infiltration zone vessels } \\
\hline $\mathrm{TAM}^{\mathrm{Iba} 1}$ & 0/7 (0) & 3/7 (43) & $4 / 7(57)$ & $0 / 9(0)$ & $6 / 9(67)$ & $3 / 9(33)$ \\
\hline $\mathrm{TAM}^{\mathrm{CD} 68}$ & $0 / 7(0)$ & $2 / 7(29)$ & $5 / 7(71)$ & $4 / 9(44)$ & $5 / 9(56)$ & $0 / 9(0)$ \\
\hline \multicolumn{7}{|c|}{ Central tumour } \\
\hline $\mathrm{TAM}^{\mathrm{Iba1}}$ & 0/7 (0) & $0 / 7(0)$ & 7/7 (100) & $0 / 9(0)$ & $2 / 9(22)$ & $7 / 9(78)$ \\
\hline $\mathrm{TAM}^{\mathrm{CD} 68}$ & $0 / 7(0)$ & $3 / 7(43)$ & $4 / 7(57)$ & $2 / 9(22)$ & $7 / 9(78)$ & $0 / 9(0)$ \\
\hline \multicolumn{7}{|c|}{ Central tumour vessels } \\
\hline $\mathrm{TAM}^{\mathrm{Iba1}}$ & 0/7 (0) & 2/7 (29) & $5 / 7(71)$ & $0 / 9(0)$ & $2 / 9(22)$ & $7 / 9(78)$ \\
\hline $\mathrm{TAM}^{\mathrm{CD} 68}$ & $0 / 7(0)$ & $2 / 7(29)$ & $5 / 7(71)$ & $0 / 9(0)$ & $4 / 9(44)$ & $5 / 9(56)$ \\
\hline \multicolumn{7}{|l|}{ Necroses } \\
\hline $\mathrm{TAM}^{\mathrm{Iba} 1}$ & 6/7 (86) & $1 / 7(14)$ & $0 / 7(0)$ & $7 / 9(78)$ & $2 / 9(22)$ & $0 / 9(0)$ \\
\hline $\mathrm{TAM}^{\mathrm{CD} 68}$ & 4/7 (57) & $3 / 7(43)$ & $0 / 7(0)$ & $8 / 9(89)$ & $1 / 9(11)$ & $0 / 9(0)$ \\
\hline
\end{tabular}

Information was also gathered concerning pseudopalisading cells that were excluded from this table due to the low number of cases. TAM, tumour-associated macrophage.

Table II. Distribution of TAMs ${ }^{\text {Ibal }}$ and $\mathrm{TAMs}^{\mathrm{CD} 68}$ at specific locations.

Comparisons of the amounts of TAMs

P-value

TAMs $^{\text {Iba1 }}$ vs. TAMs ${ }^{\mathrm{CD} 68}$ in the infiltration zone

$<0.001^{\mathrm{a}}$

$\mathrm{TAMs}^{\text {Ibal }}{ }^{\mathrm{V}}$ vs. TAMs ${ }^{\mathrm{CD} 68}$ in central tumour

$0.001^{\mathrm{a}}$

$\mathrm{TAMs}^{\mathrm{Iba} 1}$ vs. TAMs ${ }^{\mathrm{CD} 68}$ surrounding infiltration zone vessels

0.096

$\mathrm{TAMs}^{\mathrm{Ibal}}$ vs. TAMs ${ }^{\mathrm{CD} 68}$ surrounding central vessels

0.414

$\mathrm{TAMS}^{\mathrm{Ibal}}$ in the infiltration zone vs. central areas

$<0.001^{\mathrm{a}}$

TAMs $^{\text {Ibal }}$ surrounding vessels in infiltration and central areas

0.058

$\mathrm{TAMs}^{\mathrm{CD} 68}$ in the infiltration zone vs. central areas

$0.001^{\mathrm{a}}$

TAMs ${ }^{\mathrm{CD} 68}$ surrounding vessels in infiltration and central areas

0.038

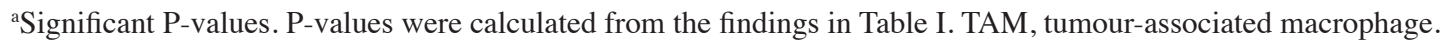

Most TAMs were located in perivascular regions and were best visualized in CD68 stained sections, especially in the infiltration zone. This may reflect the presence of TAMs in so called perivascular niches, in which there is collaboration between different cell types $(15,21,22)$. The impact of this microenvironment on the tumour biology in the periphery is unclear. However, as CD68 is highly expressed in M1 TAMs, these perivascular macrophages may provide an inhibitory effect on the infiltration process.

In the central part of the tumour, we observed a much higher number of TAMs compared with the infiltration zone, in accordance with others (9). This illustrates that these cells constitute a large part of the tumour mass, up to $30 \%(3,5)$. The TAMs were mostly diffusely dispersed; however, there was a concentration in microanatomical compartments consistent with perivascular and perinecrotic niches $(21,23)$. The rather few TAMs within necrosis are in agreement with the observation that these cells are more common in radiation-induced necrosis. This is an important feature in the differential diagnosis between tumour and radiation-induced necrosis (24), and it seems counterintuitive as this process represents a tissue stress that normally attracts TAMs (23). On the other hand, the microenvironment in these areas drive TAMs towards the M2 phenotype so they lose their ability to remove necrotic debris (21). 

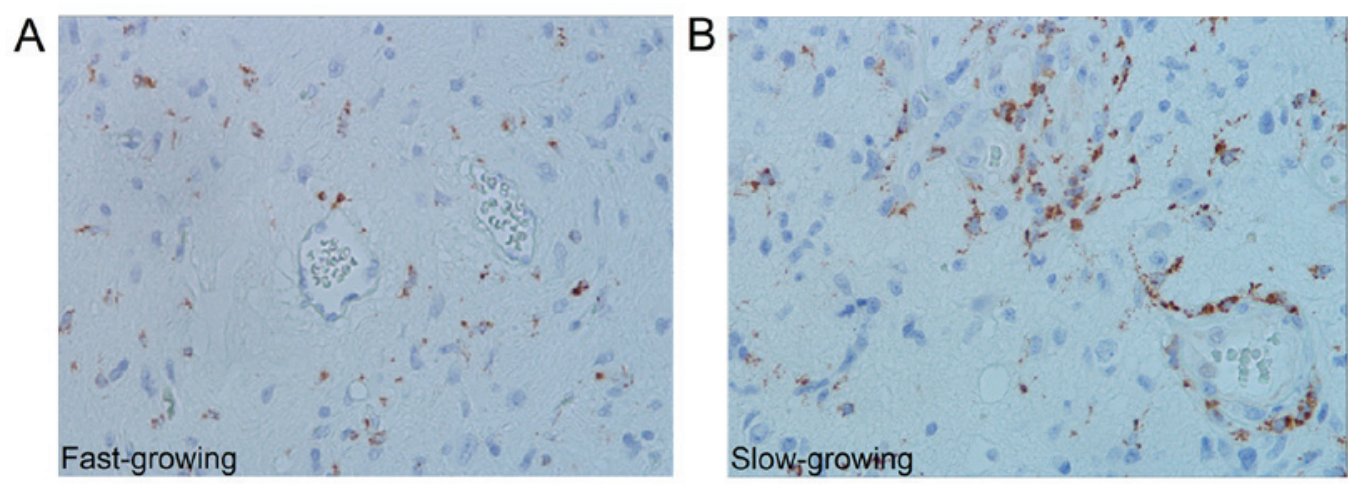

Figure 3. Increased number of TAMs ${ }^{\mathrm{CD} 68}$ surrounding peripheral vessels in slow-growing tumours. In the infiltration zone of (A) fast-growing tumours, there are relatively few labelled TAMs ${ }^{\mathrm{CD} 68}$ surrounding vessels compared with the numbers in (B) slow-growing tumours (original magnification, $\mathrm{x} 400$ ). TAMs ${ }^{\mathrm{CD} 68}$, CD68-immunoreactive tumour-associated macrophages.

Table III. Comparison of amount of TAMs in various locations in slow- and fast-growing tumours.

Amount of TAMs in slow-growing vs. fast-growing tumours at the given locations

P-value

$\mathrm{TAMs}^{\mathrm{rbal}}$ infiltration zone

1.000

TAMs $^{\text {Ibal }}$ central area

0.470

$\mathrm{TAMs}^{\mathrm{CD} 68}$ infiltration zone

0.758

$\mathrm{TAMs}^{\mathrm{CD} 68}$ central area

0.025

$\mathrm{TAMs}^{\mathrm{CD} 68}$ infiltration zone vessels

0.003

$\mathrm{TAMs}^{\text {Ibal }}$ infiltration zone vessels

0.252

TAMs $^{\text {rbal }}$ central vessels

0.837

$\mathrm{TAMs}^{\mathrm{CD} 68}$ central vessels

0.606

$\mathrm{TAMs}^{\mathrm{CD} 68}$ necrosis

0.299

$\mathrm{TAMs}^{\mathrm{Ibal}}$ necrosis

0.837

$\mathrm{TAMs}^{\mathrm{CD} 68}$ pseudopalisades

0.114

$\mathrm{TAMs}^{\mathrm{Ibal}}$ pseudopalisades

0.918

TAMs, tumour-associated macrophages.

Although this study is retrospective and based on relatively few cases, it describes histological aspects of TAMs in a series of untreated GBMs in vivo with MRI estimated tumour growth. Even though steroid treatment was administrated for many patients, this has been shown not to affect tumour growth (11). A strength of this study is that sufficient tumour tissue was available to cover the peripheral and central areas. We also used two antibodies regarded as pan-markers of macrophages/microglia to get a more complete description of TAM infiltration in the GBM tissue.

In conclusion, there is a heavy infiltration of TAMs in human GBMs, and they are commonly located in distinct microanatomical compartments, most likely constituting parts of cellular niches important for tumour biology. Both ramified and amoeboid TAMs were observed, consistent with a dynamic range of activation states throughout the tumour tissue. The number of TAMs seems less critical for tumour growth whereas the phenotype appears more relevant, pointing to a potential target for therapy that needs further investigation.

\section{Acknowledgements}

We appreciate the technical support of Unn Sophie Granli and Camilla Bjørk Setsaas. We thank Turid Follestad for statistical support.

\section{Funding}

This study was funded by the Faculty of Medicine and Health Sciences, Norwegian University of Science and Technology (grant no. 70367040).

\section{Availability of data and materials}

All data and materials used in this study is present in this manuscript.

\section{Authors' contributions}

MK performed microscopical analysis, statistical analysis and wrote the manuscript. VEM was a major contributor in writing the manuscript and in acquisition of the data. ALS and OS performed the MRI investigations and contributed in writing the manuscript. JW was involved in analysis of the data and reviewing the manuscript. SHT designed the study, supervised the results and co-wrote the manuscript.

\section{Ethics approval and consent to participate}

This project was approved by the Regional Ethics Committee (Central) as part of a larger project (references 2011/974 and 2013/1348) and adhered with the Declaration of Helsinki. The majority of patients provided written informed consent to be included in a related glioma outcome study (reference 2011/974). The regional ethics committee waived informed consent for retrospective evaluation of patient data for the remaining patients, and did not require written informed consent from a relative or guardian.

\section{Patient consent for publication}

Not applicable. 


\section{Competing interests}

The authors declare that they have no competing interests.

\section{References}

1. Louis DN, Perry A, Reifenberger G, von Deimling A, Figarella-Branger D, Cavenee WK, Ohgaki H, Wiestler OD, Kleihues P and Ellison DW: The 2016 World Health Organization Classification of Tumors of the Central Nervous System: A summary. Acta Neuropathol131: 803-820, 2016.

2. Aldape K, Zadeh G, Mansouri S, Reifenberger G and von Deimling A: Glioblastoma: Pathology, molecular mechanisms and markers. Acta Neuropathol 129: 829-848, 2015.

3. Hambardzumyan D, Gutmann DH and Kettenmann H: The role of microglia and macrophages in glioma maintenance and progression. Nat Neurosci 19: 20-27, 2016.

4. Perdiguero EG and Geissmann F: The development and maintenance of resident macrophages. Nat Immunol 17: 2-8, 2016.

5. Tremble LF, Forde PF and Soden DM: Clinical evaluation of macrophages in cancer: Role in treatment, modulation and challenges. Cancer Immunol Immunother 66: 1509-1527, 2017.

6. Domingues P, González-Tablas M, Otero Á, Pascual D, Miranda D, Ruiz L, Sousa P, Ciudad J, Gonçalves JM, Lopes MC, et al: Tumor infiltrating immune cells in gliomas and meningiomas. Brain Behav Immun 53: 1-15, 2016.

7. Li W and Graeber MB: The molecular profile of microglia under the influence of glioma. Neuro Oncol 14: 958-978, 2012.

8. Ricard C, Tchoghandjian A, Luche H, Grenot P, Figarella-Branger D, Rougon G, Malissen M and Debarbieux F: Phenotypic dynamics of microglial and monocyte-derived cells in glioblastoma-bearing mice. Sci Rep 6: 26381, 2016.

9. Boche D, Perry VH and Nicoll JA: Review: Activation patterns of microglia and their identification in the human brain. Neuropathol Appl Neurobiol 39: 3-18, 2013.

10. Colonna M and Butovsky O: Microglia function in the central nervous system during health and neurodegeneration. Annu Rev Immunol 35: 441-468, 2017.

11. Stensjøen AL, Solheim O, Kvistad KA, Håberg AK, Salvesen $\varnothing$ and Berntsen EM: Growth dynamics of untreated glioblastomas invivo. Neuro Oncol 17: 1402-1411, 2015.

12. Stensjøen AL, Berntsen EM, Mikkelsen VE, Torp SH, Jakola AS, Salvesen $\varnothing$ and Solheim O: Does pretreatment tumor growth hold prognostic information for patients with glioblastoma? World Neurosurg 101: 686-694.e4, 2017.
13. O'Malley JT, Nadol JB Jr and McKenna MJ: Anti CD163+, Iba1 ${ }^{+}$, and $\mathrm{CD} 68^{+}$cells in the adult human inner ear: Normal distribution of an unappreciated class of macrophages/microglia and implications for inflammatory otopathology in humans. Otol Neurotol 37: 99-108, 2016.

14. Zhou W, Ke SQ, Huang Z, Flavahan W, Fang X, Paul J, Wu L, Sloan AE, McLendon RE, Li X, et al: Periostin secreted by glioblastoma stem cells recruits M2 tumour-associated macrophages and promotes malignant growth. Nat Cell Biol 17: 170-182, 2015.

15. Murray PJ and Wynn TA: Protective and pathogenic functions of macrophage subsets. Nat Rev Immunol 11: 723-737, 2011.

16. Sørensen MD, Dahlrot RH, Boldt HB, Hansen S and Kristensen BW: Tumour-associated microglia/macrophages predict poor prognosis in high-grade gliomas and correlate with an aggressive tumour subtype. Neuropathol Appl Neurobiol 44: 185-206, 2018.

17. Chávez-Galán L, Olleros ML, Vesin D and Garcia I: Much more than M1 and M2 macrophages, there are also CD169(+) and TCR(+) macrophages. Front Immunol 6: 263, 2015.

18. Glass R and Synowitz M: CNS macrophages and peripheral myeloid cells in brain tumours. Acta Neuropathol 128: 347-362, 2014.

19. Mosser DM and Edwards JP: Exploring the full spectrum of macrophage activation. Nat Rev Immunol 8: 958-969, 2008.

20. Bieńkowski M and Preusser M: Prognostic role of tumour-infiltrating inflammatory cells in brain tumours: Literature review. Curr Opin Neurol 28: 647-658, 2015.

21. Hambardzumyan D and Bergers G: Glioblastoma: Defining tumor niches. Trends Cancer 1: 252-265, 2015.

22. Schiffer D, Annovazzi L, Mazzucco M and Mellai M: The microenvironment in gliomas: Phenotypic expressions. Cancers (Basel) 7: 2352-2359, 2015.

23. Yang L and Zhang Y: Tumor-associated macrophages: From basic research to clinical application. J Hematol Oncol 10: 58, 2017.

24. Prayson RA and Cohen ML (eds): Practical Differential Diagnosis in Surgical Neuropathology. Humana Press, Totowa, NJ, p177, 2000 .

This work is licensed under a Creative Commons Attribution-NonCommercial-NoDerivatives 4.0 International (CC BY-NC-ND 4.0) License. 